\title{
Fermilab Main Injector Beam Position Monitor Upgrade
}

\author{
B. Banerjee, W. Barker, S. Bledsoe, T. Boes, C. Briegel, D. Capista,
} G. Deuerling, R. Dysert, R. Forster, S. Foulkes, W. Haynes, B. Hendricks, T. Kasza, R. Kutschke, A. Marchionni, M. Olson, V. Pavlicek, L. Piccoli, P. Prieto, S. Rapisarda, A. Saewert, J. Van Bogaert, M. Votava, R. Webber, M. Wendt, N. Wilcer, S. Wolbers

Fermi National Accelerator Laboratory, Batavia, IL 60510

\begin{abstract}
An upgrade of the Beam Position Monitor (BPM) signal processing and data acquisition system for the Fermilab Main Injector is described. The Main Injector is a fast cycling synchrotron that accelerates protons or antiprotons from 8 to $150 \mathrm{GeV}$. Each Main Injector cycle can have a totally different magnet ramp, RF frequency configuration, beam bunch structure, and injection/extraction pattern from the previous cycle. The new BPM system provides the capabilities and flexibility required by the dynamic and complex machine operations. The system offers measurement capability in the $2.5 \mathrm{MHz}$ and $53 \mathrm{MHz}$ channels to detect the range of bunch structures for protons and antiprotons in both wideband (turn-by-turn) and narrowband (closed-orbit) modes. The new BPM read-out system is based on the digital receiver concept and is highly configurable, allowing the signal processing of nearly all Main Injector beam conditions, including the detection of individual batches. An overview of the BPM system in the Main Injector operating environment, some technology details and first beam measurements are presented.
\end{abstract}

\section{INTRODUCTION}

A project to upgrade the readout electronics of the Fermilab Main Injector (MI) Beam Position Monitors (BPMs) was begun in 2005. This project is one of many improvements to the Fermilab accelerator complex aimed at providing increased luminosity and reliability for the Run 2 collider physics program. The design builds upon many of the technologies and techniques used to upgrade the BPM electronics in the Recycler Ring ${ }^{1}$, the Tevatron ${ }^{2}$, as well as some of the transfer lines.

\section{OPERATIONAL REQUIREMENTS}

The Fermilab Main Injector (MI) is designed to accelerate either protons or antiprotons from an injection energy of $8 \mathrm{GeV}$ to an extraction energy as high as 150 $\mathrm{GeV}$. At any time, however, only one of the two particle species is present in the machine. 
The time structure of the beam may be one of the following:

- 53 MHz Protons. From 1 bunch up to a full batch of 84 bunches in successive $53 \mathrm{MHz}$ buckets. Up to 6 batches, 84 bunches each, can be loaded in the MI.

- $53 \mathrm{MHz}$ Antiprotons. Four consecutive groups of antiprotons spaced by 396 ns. Each group is comprised of typically 5 (up to 9) $53 \mathrm{MHz}$ bunches.

- $2.5 \mathrm{MHz}$ Protons or Antiprotons. Four bunches in successive $2.5 \mathrm{MHz}$ RF buckets (396 ns spacing).

The MI BPM system is required to be able to measure either the 2.5 or the $53 \mathrm{MHz}$ components of the beam and, in both cases, to be capable of either narrowband or wideband measurements ${ }^{3}$. Narrowband measurements, at either 2.5 or $53 \mathrm{MHz}$, have a resolution bandwidth of about $1 \mathrm{kHz}$ and effectively average signal from all bunches in the machine over approximately 40 turns. Wideband $2.5 \mathrm{MHz}$ measurements have a resolution bandwidth of about $250 \mathrm{kHz}$ and represent an average over all bunches within a window of about $1 / 28$ of the MI circumference. Wideband $53 \mathrm{MHz}$ measurements have a resolution bandwidth of about $500 \mathrm{kHz}$ and average over all bunches within a window of about $1 / 14$ of the circumference of the MI.

The narrowband mode is also referred to as closed-orbit mode. Wideband measurements, triggered to sample the same section of the circulating beam on consecutive turns, are also referred to as turn-by-turn mode.

The Main Injector functions to accept beam from one of several injection lines, accelerate the beam, perhaps change its RF structure, and deliver it to one or more extraction lines. Each distinct combination of these steps is referred to as a MI cycle. Some cycles have multiple injections, all from the same injection line. Including test and development cycles, about 20 cycle types are currently defined but new ones can be created for future operations. In typical operations a pattern of MI cycles repeats in a supercycle; the pattern may be all cycles of the same type, but typically is a mix of different cycle types. The duration of a typical cycle is 1 to 2 seconds, but some are up to 10 seconds; a supercycle is typically $60-120$ seconds

To operate effectively, the MI BPM system needs to be highly programmable with a different setup for each MI cycle type. Measurements that may be programmed in any particular cycle are described; some are mutually exclusive. Continuous narrowband closed-orbit measurements must be provided at a sustained real-time rate of at least $500 \mathrm{~Hz}$. For each injection in a cycle, wideband measurement of the newly injected beam for its first 512 turns is possible; the position on the first turn and the average position over the first 16 turns are reported separately. For one of the extractions in a cycle, a wideband measurement on the portion of the beam to be extracted, capturing the last turns (up to 512) before extraction, is possible and the last turn beam position is reported. At a specified time within the cycle, a wideband measurement of a specified part of the beam, recording the position for 2048 turns, is possible. For all of the wideband measurements, the system makes the data available at the conclusion of the cycle and retains the data until it is overwritten by another instance of the same cycle. The BPM system must be able to report synchronized wideband measurements from around the ring, with each BPM measuring the same portion of the beam. 
Finally, the MI control system periodically commands the BPM to retain the most recent narrowband measurement and to hold this measurement in a buffer for future inspection. Measurements from each cycle are kept in local memory by the front-end software system ${ }^{4}$ until overwritten by another instance of the same cycle.

The quantities that serve as the basis for the BPM measurement specifications are defined as follows:

a) Measurement range. The range of positions, relative to the BPM center, over which the BPM measurement must be valid and meet the accuracy requirements.

b) Absolute position accuracy. This determines how well the position of the beam is measured with respect to the survey center-line.

c) Relative position accuracy. This determines how well the displacement of the beam is measured over the measurement range. This requirement does not include offset errors, but does set limits on the scale errors, nonlinearities and random errors.

d) Position resolution. The smallest change in beam position that the BPM can reliably measure.

e) Position linearity. The linearity of the BPM response to orbit changes over the measurement range. The linearity is defined as the difference between the measured BPM position and the slope of the BPM response at the center of the BPM.

f) Long-term position stability. The BPM systems ability to give the same position value for the same beam position and intensity over time.

g) Relative intensity accuracy. The relative intensity measurements between BPMs.

Table 1 summarizes measurement specifications for $53 \mathrm{MHz}$ and $2.5 \mathrm{MHz}$ bunch structures, respectively.

\begin{tabular}{|c|c|c|}
\hline $\begin{array}{l}\text { Measurement } \\
\text { specifications }\end{array}$ & $\begin{array}{l}2.5 \mathrm{MHz} \text { bunch structure } \\
\text { (anti-protons) }\end{array}$ & $53 \mathrm{MHz}$ bunch structure \\
\hline Measurement range & $\pm 25 \mathrm{~mm}$ & $\pm 25 \mathrm{~mm}$ \\
\hline $\begin{array}{l}\text { Absolute position } \\
\text { accuracy }\end{array}$ & $1 \mathrm{~mm}+10 \%$ of actual beam position & $1 \mathrm{~mm}+5 \%$ of actual beam position \\
\hline $\begin{array}{c}\text { Relative position } \\
\text { accuracy }(3 \sigma)\end{array}$ & $10 \%$ of actual beam position & $5 \%$ of actual beam position \\
\hline $\begin{array}{l}\text { Position resolution } \\
\qquad(3 \sigma)\end{array}$ & $0.3 \mathrm{~mm}(0.5 \mathrm{~mm})$ & $0.05 \mathrm{~mm}(0.1 \mathrm{~mm})$ \\
\hline $\begin{array}{l}\text { Long-term position } \\
\text { stability }\end{array}$ & $0.5 \mathrm{~mm}$ & $0.2 \mathrm{~mm}$ \\
\hline $\begin{array}{c}\text { Relative intensity } \\
\text { accuracy }\end{array}$ & $20 \%(30 \%)$ & $10 \%(20 \%)$ \\
\hline
\end{tabular}

Table 1: Measurement specifications for $2.5 \mathrm{MHz}$ and $53 \mathrm{MHz}$ bunch structures in narrow bandwidth mode (values in parenthesis are single turn resolution for high bandwidth mode). The requirements are stated for nominal bunch intensities; relaxed requirements are permitted for low intensity bunches. 


\section{THE MI-BPM READ-OUT ELECTRONICS HARDWARE}

Figure 1 gives an overview of the hardware components of the upgraded Main Injector BPM system. The figure shows two signal channels that produce one position measurement channel. The 201 stripline BPM pickups and the tunnel cabling are the original Main Injector components; they remain unchanged ${ }^{5}$. Upgraded components are the combiner boxes, located in the accelerator tunnel, and all read-out electronics hardware, concentrated in seven locations around the ring. The analog electronics, 8 -channel transition modules and a controller module, are located in a $6 \mathrm{U}$ high crate frame, supplied by an external, linear power supply. A core component of the BPM system is the commercial Echotek GC814 digital signal receiver. Several 8-channel receivers, together with a processor module (Motorola MVME5500) and an in-house developed timing \& fan-out generator (TFG-II), are arranged in a $6 \mathrm{U}$ VME crate. All the cabling within a rack and between crates is new.

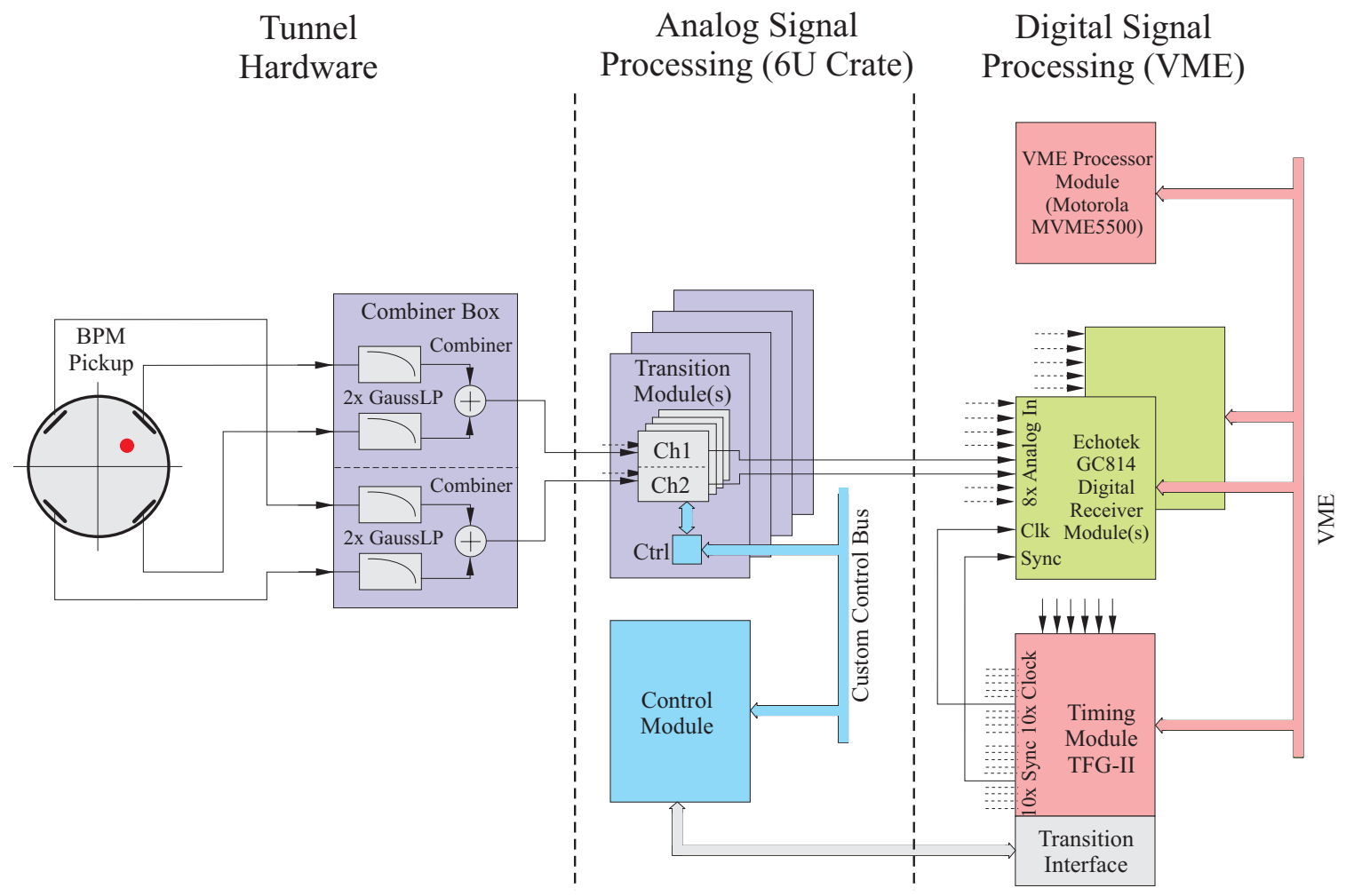

Figure . Overview of the Main Injector BPM hardware, showing 2 of 430 channels total. The Combiner Box is shown as configured to make a horizontal measurement.

\section{Timing and Trigger Distribution}

The timing and fan-out generator (TGF-II) is a double width, 6U VME card that is an evolution of the Tevatron TGF card ${ }^{2}$. The TGF-II receives cycle timing and control signals from the accelerator control system and beam synchronous information from the MI, the Recycler and the Booster accelerators. These are translated into the timing 
and control signals required for the operation of the BPM system, including those signals required by the VME processor, the transition modules, and the digital receivers. TGF-II is also the source for diagnostic signals that can be injected into the RF signal path on the transition boards.

A fundamental timing signal utilized by the TGF-II is the MI RF signal, the master beam synchronous timing reference for the MI. The TGF-II card uses this reference to generate the digitization clock at 10/7 of the RF frequency which it distributes to each digital receiver board. The digitization clock tracks all changes in the MI RF frequency, ensuring that the pass-band of each digital downconverter (DDC) channel remains centered on the signal frequency.

\section{Analog Signal Pre-Processing System}

Analog signal processing occurs in both the combiner boxes and the transition modules. The combiner boxes accept beam signals from the four stripline electrodes, pass them through a low-pass filter $\left(f_{\mathrm{c}} \approx 100 \mathrm{MHz}\right)$, and combine them to form either a vertical measuring or a horizontal measuring signal pair. The analog transition modules interface the signals from the combiner boxes to the digital receivers, adapting signal levels and further filtering frequency content.

The broadband signals from the BPM pickups require analog frequency-domain filtering and amplitude management before presentation to the digital receivers. Therefore each transition module channel is actually comprised of two parallel signal paths, one for the $2.5 \mathrm{MHz}$ signal band and another for the $53 \mathrm{MHz}$ band, each with appropriate bandwidth shaping filters and programmable gain. The bandwidth of each is about $5 \mathrm{MHz}$, chosen to allow resolution of individual batches in the Main Injector. All signal paths have remote controlled features, including enable/disable, gain control (range: $0 \ldots 48 \mathrm{~dB}$ ), and low/high post-amplification switch.

Test signals of 2.5 or $53 \mathrm{MHz}$, supplied by the TGF-II module, can be applied to the transition module inputs to simulate centered or displaced beam. This test signal capability, as well as all remote operations is controlled through local programmable logic. The central control of an analog crate is managed by a control module that is connected to the VME module through a rear transition board (Figure 1). Further details on the analog pre-processing electronics are presented elsewhere in these proceedings $^{6}$.

\section{Digital Signal Processing System}

Eight-channel Echotek ECDR-GC814-FV2 VME64X digital receiver boards are used for beam signal digitization and frequency conversion. Each GC814 channel is comprised of a 14 bit ADC (AD6645), a four-channel DDC (GC-4016), an FPGA accumulator, and $128 \mathrm{~K} \times 32$ dual-ported RAM. These boards shift the information content of the beam signal from a RF analog form to a stream of digital, baseband I and $\mathrm{Q}$ data pairs. Every BPM utilizes two receiver channels, referred to as channels A and $\mathrm{B}$. 
The position, $\mathrm{P}$, and the intensity, I, are given by:

$$
\begin{aligned}
& A=\sqrt{I_{A}+Q_{A}}, \quad B=\sqrt{I_{B}+Q_{B}}, \quad R=\frac{A-B}{A+B}, \\
& P=a_{0}+a_{1} R+a_{3} R^{3}+a_{5} R^{5}, \quad I=A+B,
\end{aligned}
$$

where the polynomial coefficients were determined from bench test measurements of the pickups.

The $53 \mathrm{MHz}$ frequency is harmonic 588 of the MI beam revolution frequency and the 53 and $2.5 \mathrm{MHz}$ beam frequencies are harmonically related in the ratio of 21:1. By design choice, the ADC clock $\left(\mathrm{f}_{\mathrm{ADC}}\right)$ is locked to $10 / 7$ times the $53 \mathrm{MHz}$ frequency. This is approximately $76 \mathrm{MHz}$ with a $400 \mathrm{kHz}$ sweep during acceleration. Given this digitization frequency, the $2.5 \mathrm{MHz}$ beam signal band is oversampled. The $53 \mathrm{MHz}$ signal band, however, is undersampled by the digitizer and aliased down to $3 / 10 \mathrm{f}_{\mathrm{ADC}}$, about 22.8 MHz. The $53 \mathrm{MHz}$ analog bandpass filters on the transition board suppress unwanted images. Digitized signals are down-converted to baseband in the GC-4016 with numerically controlled local oscillators operating at $3 / 10 \mathrm{f}_{\mathrm{ADC}}$ for the $53 \mathrm{MHz}$ channels and $1 / 30 \mathrm{f}_{\mathrm{ADC}}$ for the $2.5 \mathrm{MHz}$ channels.

Figure 2 is a block diagram of one of four channels in each GC-4016 digital downconverter. All four channels of each GC-4016 DDC are utilized. Channels 1 and 2 are configured for 53 and $2.5 \mathrm{MHz}$ narrowband operation respectively and channels 3 and 4 for wideband operation.

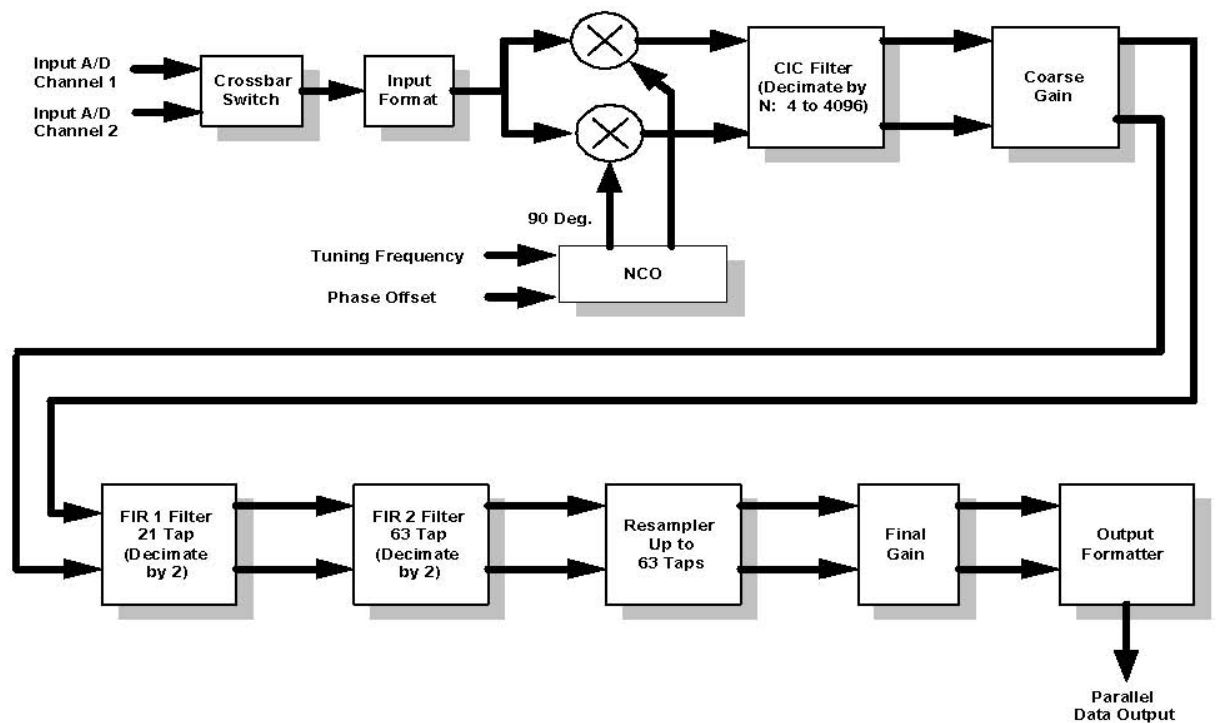

FIGURE 2. Block diagram of one of four channels in each GC-4016 digital downconverter IC.

Narrowband filtering is achieved with decimation of 1536 in the 5-stage CIC filter and decimation of two in each of the CFIR (FIR 1) and PFIR (FIR 2) filters. For $\mathrm{f}_{\mathrm{ADC}}=75.86 \mathrm{MHz}$, the CIC output update and CFIR tap interval is 20.2 microseconds. The PFIR tap interval is $\sim 40.5 \mu$ s and its output updates at $\sim 81.0 \mu \mathrm{s}$. Each narrowband CFIR is set up as an 11-tap Gaussian filter and each PFIR as an 11-tap boxcar filter. 
The eleven taps in the PFIR correspond to a signal integration window of $445 \mu \mathrm{s}(11 \mathrm{x}$ $40.5 \mu \mathrm{s}$ ), about $40 \mathrm{MI}$ turns. The $3 \mathrm{db}$ bandwidth of the narrowband channels is $1 \mathrm{KHz}$.

Wideband channels, both 2.5 and $53 \mathrm{MHz}$, are programmed to have a total decimation of 32, distributed as 8 in the CIC filter and 2 each in the CFIR and PFIR filters. With a $75.86 \mathrm{MHz}$ digitizer frequency, the sampling interval is 106 nanoseconds in the CFIR, $\sim 211 \mathrm{~ns}$ in the PFIR and $\sim 422 \mathrm{~ns}$ out of the PFIR. The wideband CFIR and PFIR are both configured as boxcar filters.

The $53 \mathrm{MHz}$ wideband channel CFIR has just two non-zero coefficients and the PFIR has four, producing a signal integration time of $844 \mathrm{~ns}(4 \times 211 \mathrm{~ns})$, about $1 / 2$ of one MI beam batch. This window is a compromise to include signal from half of one batch while providing good isolation from signals due to adjacent batches. The resulting effective bandwidth of the $53 \mathrm{MHz}$ wide-band channel is about $500 \mathrm{KHz}$.

The $2.5 \mathrm{MHz}$ wideband channel CFIRs also have two non-zero coefficients; the PFIR has eight taps providing a signal integration window of $1687 \mathrm{~ns}$ ( 8 × $211 \mathrm{~ns})$. This window is sufficient to include signal from all four sequential $2.5 \mathrm{MHz}$ bunches, typical of the beam structures for which this channel is used. The effective bandwidth of the $2.5 \mathrm{MHz}$ wide-band channel is $250 \mathrm{KHz}$.

\section{PERFORMANCE}

Approximately two weeks before the spring 2006 Fermilab accelerator shutdown a pre-production system was installed to read out 11 BPMs. The results presented in this section were obtained using this pre-production system.

Figure 3 illustrates the narrow band capability for an MI cycle in which six batches are injected from the Booster, accelerated to $120 \mathrm{GeV}$, moved onto the extraction orbit and delivered to the neutrino production target. The six injections occur at intervals of $1 / 15$ second and the beam is extracted in a single turn.

Part a) of Figure 3 shows the electrode sum, $A+B$, as a function of time. For constant machine energy the electrode sum is proportional to the beam intensity and the six injections can be seen. The modulation in $\mathrm{A}+\mathrm{B}$ after the sixth injection is due to changes in the bunch length as the beam energy is increased. Part b) of Figure 3 shows the measured position for this same dataset.

For the last 12 ticks (48 ms) before extraction, the beam position is reasonably constant on the extraction orbit. The RMS spread of measured positions during this interval for the six instrumented vertical measuring BPMs is typically 2.5 microns. The predicted resolution for the system is less than 1 micron so it is believed that this RMS is dominated by true beam motion. At the five horizontal measuring locations, there is obvious beam motion that is folded into the measured RMS values, which range from about 5 to 10 microns. In all cases these RMS values, even uncorrected for true beam motion, are well below the required resolution of 17 microns ( 1 sigma).

The pre-production system has also allowed a measurement of the stability of the MI. Narrowband measurements were made for 281 cycles over a period of a half hour. At each BPM the mean orbit and the RMS spread about the mean orbit were computed. At most BPMs, at most times in the cycle, the RMS spread of the measured position is less than 10 microns. Even at the least stable locations the spread was less 
than 50 microns for most times in the cycle. This represents the most precise determination to date of the stability of the MI.

The data in Figure 3 can also be used to illustrate the flexibility of the upgraded BPM system. The system can, for example, be programmed to make turn-by-turn measurements of the newly injected batch at each of the 7 injections, to make closedorbit measurements during the middle of the cycle, and to make turn-by-turn measurements of any one batch at its extraction. This has been demonstrated but the data are not shown here.

Figure 4 shows a wideband $2.5 \mathrm{MHz}$ measurement for a cycle that takes antiprotons from the Recycler and injects them into the Tevatron. Parts a) and b) of the figure show, respectively, the electrode sum and the position. The measurement was triggered about 28 turns before the beam was injected so the first 28 points illustrate the low noise level of the system. Part c) shows the Fourier transform of the position data, in which a strong betatron line can be seen.
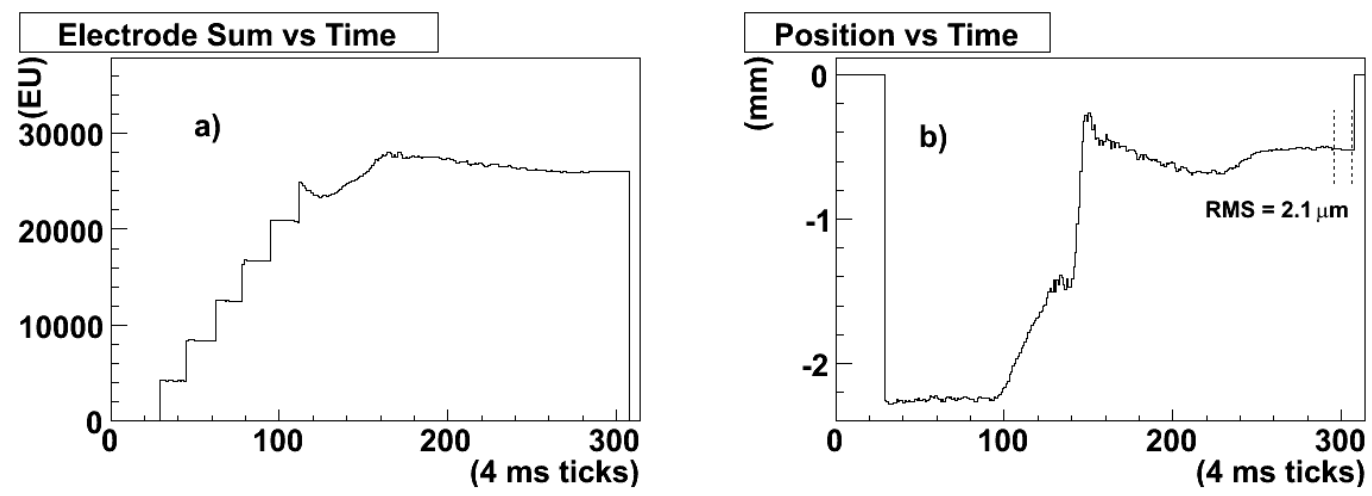

Figure 3. Part a) shows the electrode sum $A+B$, for a vertical measuring BPM for a MI cycle as described in the text. For both parts of this figure, the horizontal axis is time, measured in $4 \mathrm{~ms}$ ticks; the system can deliver data with 2 ms ticks but this dataset was taken at the slower rate. The noise level in the electrode sum is not visible on this scale. Part b) shows the measured vertical position for the same dataset. The dashed vertical lines near the right edge of the plot mark 12 measurements used to estimate the resolution of the system. The RMS spread of these 12 points is 2.1 microns.
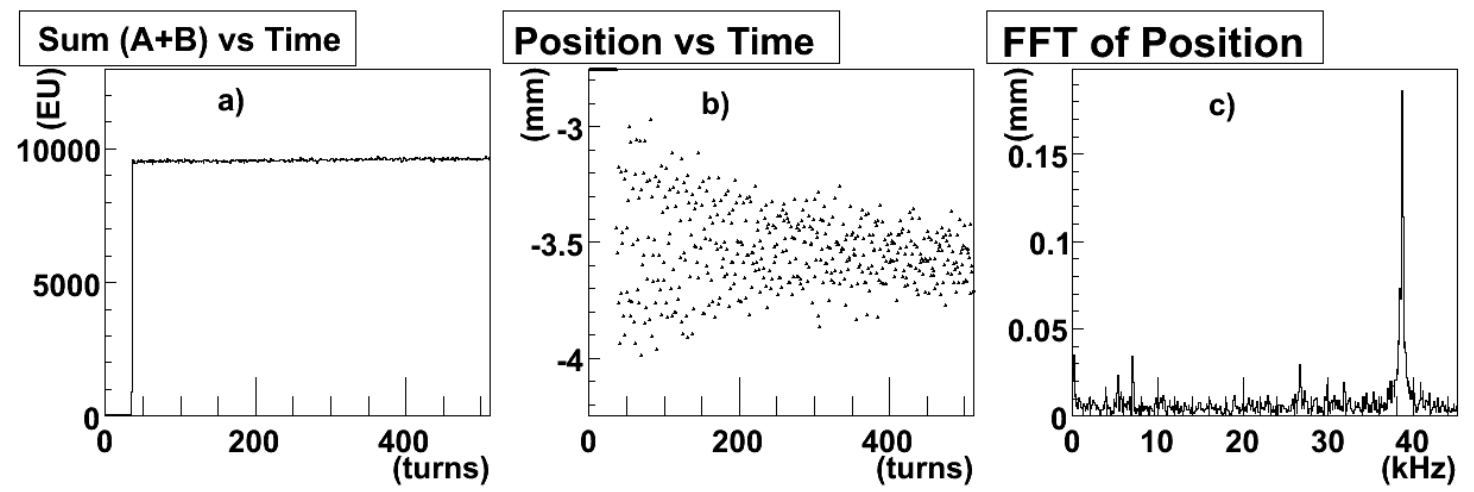

Figure 4. Part a) shows the electrode sum for a turn-by-turn measurement of anti-protons injected from the Recycler. The noise level is barely visible on this scale. Part b) shows the measured position. For parts a) and b) the horizontal axis is turn number. The FFT of the position data is shown in part c); the mean position was subtracted before computing the FFT. The betatron tune line is strong. 


\section{CONCLUSION}

An upgrade to the Fermilab Main Injector Beam Position Monitor electronics and readout has been designed and prototyped. Initial measurements with the prototype system show performance that meets or exceeds the requirements for machine operations. Final hardware and software is being prepared for final installation and commissioning in the summer of 2006.

\section{ACKNOWLEDGMENTS}

This work was supported by Fermi National Accelerator Laboratory, operated by Universities Research Association Inc. under Contract No. DE-AC02-76CH03000 with the United States Department of Energy.

\section{REFERENCES}

${ }^{1}$ Webber, R. et. al. "FermiLab Recycler Ring BPM Upgrade Based on Digital Receiver Technology" Beam Instrumentation Workshop 2004, Knoxville, Tennessee 3-6 May 2004.

${ }^{2}$ S. Wolbers, et al., "Tevatron Beam Position Monitor Upgrade”, PAC2005, Knoxville, TN, 16-20 May 2005.

${ }^{3}$ D. Capista, et al., "Requirements for the Main Injector BPM Upgrade", https://beamdocs. fnal.gov/AD-public/DocDB/ShowDocument?docid=1786

${ }^{4}$ L. Piccoli, et al., "The Main Injector Beam Position Monitor Front-End Software”, submitted to Beams Instrumentation Workshop, Fermilab, Batavia, IL, 1-4 May 2006.

${ }^{5}$ Fitzgerald, J., Crisp, J. "A Compact BPM for the Fermilab Main Injector" IEEE PAC, 97CH36167, May 1997.

${ }^{6}$ M. Wendt, et al., "Analog Signal Pre-Processing for the Fermilab Main Injector BPM Upgrade”, submitted to Beams Instrumentation Workshop, Fermilab, Batavia, IL, 1-4 May 2006. 